\title{
NETWORKED CONTROL SYSTEMS DESIGN WITH TIME-VARYING DELAYS
}

\author{
Ho-Jun Yoo* and Oh-Kyu Kwon ${ }^{* *, 1}$ \\ * Samsung Electronics Co. Ltd, Osan, Korea \\ ** School of Electrical Engr., Inha Univ., Incheon, Korea
}

\begin{abstract}
This paper has proposed a controller design method for networked control systems with network induced delays by using LMI-based delay-dependent optimization. The delays are described by a Markov chain, since they are to be time-varying and even longer than the sampling time. A delay-dependent compensation method has been proposed here with a jump linear system analysis technique to stabilize the networked control system. To exemplify the performance of the proposed method, some simulations applied to numerical examples with the networked control are performed. (C) Copyright 2005 IFAC
\end{abstract}

Keywords: Networked control system, delay compensation, time-varying delay, jumped linear system, linear matrix inequality.

\section{INTRODUCTION}

Recently network systems are widely used in several areas, and some considerable attentions have been directed to the Networked Control System (NCS). The NCS is defined as a feedback control system that the control loops are closed through a real-time network. In NCS, some network induced-delays occur inevitably to degrade the system dynamic performance, and even to be a source of potential instability.

The network induced-delays are time-varying and possibly have stochastic traffic property as well as mis-synchronization between control system components and noise in the communication medium. By making the buffers longer than the worst case delay, the network induced delay can be regarded as time-invariant (Luck and Ray, 1990).

\footnotetext{
1 All correspondence should be addressed to e-mail: okkwon@inha.ac.kr. This work was supported by KESRI(R-2003-B-425), which is funded by MOCIE (Ministry of Commerce, Industry and Energy).
}

The stability and performance analyses of NCS are performed in several literatures. However, the compensator design of NCS are still on beginning steps. In (Zhang et al., 2001), a current-state estimator method is proposed to compensate the stochastic network induced delay. In (Nilsson and Bernhardsson, 1997) and (Yoo et al., 2002), some compensation methods are presented for NCS. However, these methods have a limitation since they have considered only the case that the total maximum network induced delay is shorter than one sampling period.

In the current paper, an LMI-based delay-dependent optimization for time-varying delayed NCS is proposed to overcome the limitation of existing methods. The time-varying delays are described by a Markov chain which can be solved by the jumped linear system approach (Mariton, 1990; Boukas and Liu, 2001). The control system analysis and design proposed here are based on a delaydependent stabilization, and account for the size of delays. Therefore the proposed compensation method can be applied to the networked control systems with time-varying delays even longer than 
the sampling period, and that is the main contribution of the current paper.

\section{NCS DESCRIPTION}

\subsection{NCS structure with network induced delays}

The NCS with the network induced delay can be depicted by a block diagram in Fig.1. In the figure, the whole closed-loop system consists of a continuous-time plant and a discrete-time controller. The setup has considered that time-driven sensors sample the plant outputs periodically at the sampling instant; an event-driven controller and actuator are to be implemented by an external event interrupt mechanism which calculates the signal as soon as the data arrives. In the NCS description, it is assumed that the continuoustime plant is linear time-invariant and given as follows:

$$
\dot{x}(t)=A x(t)+B u(t),
$$

and the discrete-time controller is taken as a constant state feedback control as follows:

$$
u(k)=K x(k),
$$

where $x \in \Re^{n}, u \in \Re^{m}$, and $A, B$, and $K$ are of compatible dimensions.

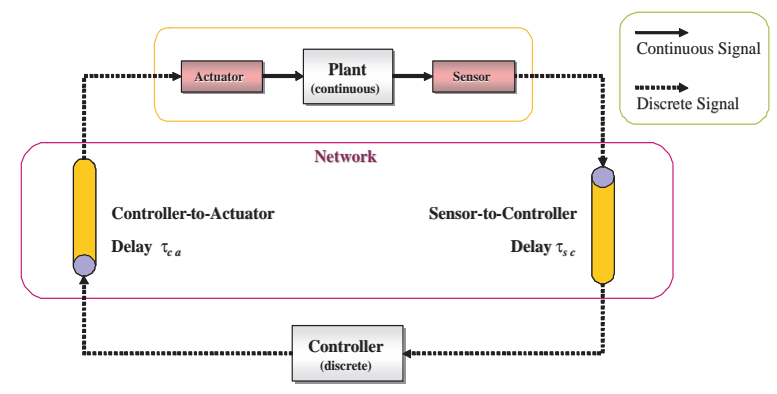

Fig. 1. NCS configuration

There are two sources of delays from the network: One is sensor-to-controller delay $\tau_{s c}$ and another is controller-to-actuator delay $\tau_{c a}$. For timeinvariant controllers, they can be lumped together as $\tau=\tau_{s c}+\tau_{c a}$ (Halevi and Ray, 1988). In our previous work (Yoo et al., 2002), the network delay $\tau$ is made time-invariant by introducing buffers at the controller and actuator nodes. Although the constant delay is a convenient form to describe the network induced delay, the buffer system has some shortcoming in that it makes the delay become longer than necessary, which makes the system performance worse. Thus, in this paper, we assume that the network induced delay has timevarying properties described by Markov chains.

If the delay $\tau_{k}$ is longer than sampling period $h$ at each $k$ step, then it can be separated into two parts as follows:

$$
\tau_{k}=\left(d_{k}-1\right) h+\tau_{k}^{\prime}, \quad 0<\tau_{k}^{\prime} \leq h,
$$

where $d_{k} \geq 2$ is an integer parameter that depends on Markov state at $k$ th step. The finite state discrete Markov process has following Markov properties:

$$
\mathbb{P}\left\{\tau_{k+1}=j \mid \tau_{k}=i\right\}=p_{i j}, \quad 0 \leq i, j \leq N,
$$

where $\mathbb{P}$ is the probability and $N$ is the number of Markov states. Then the networked closed-loop system with Markov-stated delay can be rewritten as follows:

$$
\begin{aligned}
u\left(t^{+}\right) & =K\left(d_{k}\right) x\left(t-\tau_{k}\right) \\
\dot{x}(t) & =A x(t)+B u\left(t^{+}\right) \\
& =A x(t)+B K\left(d_{k}\right) x\left(t-\tau_{k}\right),
\end{aligned}
$$

where $K\left(d_{k}\right)$ is a static memoryless controller gain for each of Markov state, and $u\left(t^{+}\right)$is piecewise continuous and changes its value only at $k+\tau_{k}$.

\subsection{State augmentation of NCS}

As sampling the networked closed-loop system (4)-(5) with the period $h$, the discrete-time linear system with the time delay (3) is obtained as follows (Åström, 1997):

$$
\begin{aligned}
x(k+1)= & \Phi x(k)+\Gamma_{0}\left(\tau_{k}^{\prime}\right) K\left(d_{k}\right) x\left(k-d_{k}+1\right) \\
& +\Gamma_{1}\left(\tau_{k}^{\prime}\right) K\left(d_{k}\right) x\left(k-d_{k}\right),
\end{aligned}
$$

where $x(k)=0$ for $k<0, x(0)=x_{0}, \Phi=e^{A h}$, and

$$
\Gamma_{0}\left(\tau_{k}^{\prime}\right)=\int_{0}^{h-\tau_{k}^{\prime}} e^{A s} B d s, \Gamma_{1}\left(\tau_{k}^{\prime}\right)=\int_{h-\tau_{k}^{\prime}}^{h} e^{A s} B d s .(7)
$$

In the sampled system (6), those two delayed states have a one-step difference, so they can be augmented into a new state vector as follows:

$$
X(k) \equiv\left[\begin{array}{c}
x(k) \\
x(k-1)
\end{array}\right] .
$$

Then the augmented system is represented by

$$
X(k+1)=\tilde{\Phi} X(k)+\tilde{\Gamma}\left(d_{k}, \tau_{k}^{\prime}\right) X\left(k-d_{k}+1\right),
$$

where

$$
\begin{aligned}
\tilde{\Phi} & =\left[\begin{array}{cc}
\Phi & 0 \\
I_{n \times n} & 0
\end{array}\right] \\
\tilde{\Gamma}\left(d_{k}, \tau_{k}^{\prime}\right) & =\left[\begin{array}{cc}
\Gamma_{0}\left(\tau_{k}^{\prime}\right) K\left(d_{k}\right) & \Gamma_{1}\left(\tau_{k}^{\prime}\right) K\left(d_{k}\right) \\
0 & 0
\end{array}\right] .
\end{aligned}
$$

From now on, the above augmented network control system is to be handled in the feedback stabilization techniques. 


\section{NCS CONTROLLER DESIGN}

Firstly, assuming that time-varying discretized input matrices $\Gamma_{0}\left(\tau_{k}^{\prime}\right)$ and $\Gamma_{1}\left(\tau_{k}^{\prime}\right)$ in Eq. (7) can be regarded as time invariant, i.e.,

$$
\Gamma_{0}\left(\tau_{k}^{\prime}\right) \equiv \Gamma_{0} \quad \text { and } \quad \Gamma_{1}\left(\tau_{k}^{\prime}\right) \equiv \Gamma_{1},
$$

the augmented input matrix (9) can be rewritten as follows:

$$
\tilde{\Gamma}\left(d_{k}, \tau_{k}^{\prime}\right)=\tilde{\Gamma}\left(d_{k}\right) .
$$

This assumption is for the sake of convenience to solve the problem. In this case, we can regard the time-varying delay property as integer valued Markov chains, but our formulation can be expanded to the case without this assumption, which will be shown later.

Now let us summarize a lemma, which plays an important role to obtain the delay dependent stabilization condition, as follows:

Lemma 1. (Lee et al., 2001) Assume that $\alpha(\cdot) \in$ $\Re^{n_{a}}, \beta(\cdot) \in \Re^{n_{b}}$, and $\mathcal{N}(\cdot) \in \Re^{n_{a} \times n_{b}}$ are defined on an interval $\Omega$. Then, for any matrices $\mathcal{X} \in$ $\Re^{n_{a} \times n_{a}}, \mathcal{Y} \in \Re^{n_{a} \times n_{b}}$, and $\mathcal{Z} \in \Re^{n_{b} \times n_{b}}$ satisfying

$$
\left[\begin{array}{ll}
\mathcal{X} & \mathcal{Y} \\
\mathcal{Y}^{T} & \mathcal{Z}
\end{array}\right] \geq 0
$$

the following inequality holds:

$$
-2 \alpha^{T} \mathcal{N} \beta \leq \inf _{\mathcal{X}, \mathcal{Y}, \mathcal{Z}}\left[\begin{array}{l}
\alpha \\
\beta
\end{array}\right]^{T}\left[\begin{array}{cc}
\mathcal{X} & \mathcal{Y}-\mathcal{N} \\
\mathcal{Y}^{T}-\mathcal{N}^{T} & \mathcal{Z}
\end{array}\right]\left[\begin{array}{l}
\alpha \\
\beta
\end{array}\right](12)
$$

Let us define the variable $K\left(d_{k}\right)$ for each Markov state as $K\left(d_{k}\right) \equiv K(i)$ for $d_{k}=i$. Then the following theorem presents a sufficient condition to assure the asymptotic stability for augmented closed-loop NCS (8) with assumption (10)-(11). This theorem is to be induced by the delay dependent stabilization technique and the Markovian jump linear system control method (Boukas and Liu, 2001).

Theorem 2. Suppose that there exist $P(i)>$ $0, K(i)(i=1, \ldots, N), Q \mathcal{X}, \mathcal{Y}$, and $\mathcal{Z}$ such that

$$
\left[\begin{array}{cccc}
\Sigma_{11} & -\mathcal{Y} & \tilde{\Phi}^{T} & \Sigma_{41}^{T} \\
-\mathcal{Y}^{T} & -Q & \tilde{\Gamma}^{T}\left(d_{k}\right) & \Sigma_{42}^{T} \\
\tilde{\Phi} & \tilde{\Gamma}\left(d_{k}\right) & -\bar{P}^{-1} & 0 \\
\Sigma_{41} & \Sigma_{42} & 0 & -(\bar{d}-1) \mathcal{Z}^{-1}
\end{array}\right]<0
$$

and

$$
\left[\begin{array}{cc}
\mathcal{X} & \mathcal{Y} \\
\mathcal{Y}^{T} & \mathcal{Z}
\end{array}\right] \geq 0
$$

where

$$
\begin{gathered}
\Sigma_{11}=-P(i)+\left(d_{k}-1\right) \mathcal{X}+\mathcal{Y}+\mathcal{Y}^{T}+\rho Q \\
\Sigma_{41}=(\bar{d}-1)\left(\tilde{\Phi}-I_{2 n \times 2 n}\right), \quad \Sigma_{42}=(\bar{d}-1) \tilde{\Gamma}\left(d_{k}\right) \\
\rho=1+(\bar{d}-\underline{d})(1-\underline{p}) \\
\bar{d}=\max \{d(i), i=1,2, \ldots, N\} \\
\underline{d}=\min \{d(i), i=1,2, \ldots, N\} \\
\underline{p}=\min \left\{p_{i i}, i=1,2, \ldots, N\right\} \\
\bar{P}=\sum_{j=1}^{N} p_{i j} P(j) .
\end{gathered}
$$

Then the closed-loop system (6) with assumption (10)-(11) is asymptotically stable for the timevarying network induced delay $\tau_{k}$ satisfying $0 \leq$ $\tau_{k} \leq\left(d_{k}-1\right) h+\tau^{\prime}$, and $\tau^{\prime}$ is constant value with $0<\tau^{\prime} \leq h$, where $h$ is the sampling period, and $d_{k} \geq 1$ is the integer valued Markov state.

Proof : Since it holds that

$$
x\left(k-d_{k}+1\right)=x(k)-\sum_{j=k-d_{k}+2}^{k}[x(j)-x(j-1)],
$$

the closed-loop NCS (6) can be represented by the augmented form as follows:

$$
\begin{aligned}
X(k+1) & =\left[\tilde{\Phi}+\tilde{\Gamma}\left(d_{k}\right)\right] X(k) \\
& -\tilde{\Gamma}\left(d_{k}\right) \sum_{j=k-d_{k}+2}^{k}[X(j)-X(j-1)] .
\end{aligned}
$$

Next, let us define a Lyapunov functional candidate as follows:

$$
\begin{aligned}
V\left(X_{k}, d_{k}\right) \equiv & V_{1}\left(X_{k}, d_{k}\right)+V_{2}\left(X_{k}, d_{k}\right) \\
& +V_{3}\left(X_{k}, d_{k}\right)+V_{4}\left(X_{k}, d_{k}\right),
\end{aligned}
$$

where

$$
\begin{aligned}
V_{1}\left(X_{k}, d_{k}\right) & \equiv X^{T}(k) P(k) X(k) \\
V_{2}\left(X_{k}, d_{k}\right) \equiv & \sum_{i=-\bar{d} j=k+i+2}^{-2} \sum^{k}[X(j)-X(j-1)]^{T} \\
& \times \mathcal{Z}[X(j)-X(j-1)] \\
V_{3}\left(X_{k}, d_{k}\right) \equiv & \sum_{j=k-d_{k}+1}^{k-1} X^{T}(j) Q X(j) \\
V_{4}\left(X_{k}, d_{k}\right) \equiv & \sum_{\theta=-\bar{d}+2}^{-\underline{d}+1} \sum_{l=k+\theta}^{k-1}(1-\underline{p}) X^{T}(l) Q X(l) .
\end{aligned}
$$

Taking $\alpha(\cdot), \beta(\cdot)$, and $\mathcal{N}$ in Lemma 1 as follows:

$$
\begin{aligned}
\alpha & \equiv X(k), \beta \equiv X(j)-X(j-1) \\
\mathcal{N} & \equiv\left[\tilde{\Phi}+\tilde{\Gamma}\left(d_{k}\right)\right]^{T} \bar{P}(i) \tilde{\Gamma}\left(d_{k}\right),
\end{aligned}
$$

and applying Lemma 1 here, it can be shown that the whole increment of the Lyapunov functional candidate (15) satisfies the following inequality: 
$\Delta V\left(X_{k}, d_{k}\right)$

$$
\begin{aligned}
& =\Delta V_{1}\left(X_{k}, d_{k}\right)+\Delta V_{2}\left(X_{k}, d_{k}\right)+\Delta V_{3}\left(X_{k}, d_{k}\right)+\Delta V_{4}\left(X_{k}, d_{k}\right) \\
& \leq\left[\begin{array}{c}
X(k) \\
X\left(k-d_{k}+1\right)
\end{array}\right]^{T} H\left[\begin{array}{c}
X(k) \\
X\left(k-d_{k}+1\right)
\end{array}\right]<0,
\end{aligned}
$$

where

$$
\begin{aligned}
H= & {\left[\begin{array}{ll}
H_{11} & H_{12} \\
H_{12}^{T} & H_{22}
\end{array}\right] } \\
H_{11}= & \tilde{\Phi}^{T} \bar{P}(i) \tilde{\Phi}-P(i)+\left(d_{k}-1\right) \mathcal{X}+\mathcal{Y}+\mathcal{Y}^{T} \\
& +(\bar{d}-1)(\tilde{\Phi}-I)^{T} \mathcal{Z}(\tilde{\Phi}-I)+\{1+(\bar{d}-\underline{d})(1-\underline{p})\} Q \\
H_{12}= & -\mathcal{Y}+\tilde{\Phi}^{T} \bar{P}(i) \tilde{\Gamma}\left(d_{k}\right)+(\bar{d}-1)(\tilde{\Phi}-I)^{T} \mathcal{Z} \tilde{\Gamma}\left(d_{k}\right) \\
H_{22}= & \tilde{\Gamma}^{T}\left(d_{k}\right) \bar{P}(i) \tilde{\Gamma}\left(d_{k}\right)+(\bar{d}-1) \tilde{\Gamma}^{T}\left(d_{k}\right) \mathcal{Z} \tilde{\Gamma}\left(d_{k}\right)-Q .
\end{aligned}
$$

It follows from the Schur complements (Boyd et al., 1994) and some change of variables that (13) is equivalent to the negative definiteness of $H$. Therefore, from Lyapunov-Krasovskii stability theorem, it follows that the closed-loop system (6) is asymptotically stable if (13) and (14) hold.

Next, without previous assumptions (10)-(11), we will derive a sufficient condition for the asymptotical stability of the NCS having time-varying delays. In this case, the small time-varying delay parts influenced by $\tau_{k}^{\prime}$ in $\Gamma_{0}$ and $\Gamma_{1}$ are regarded as the uncertainty structures, and the discretized input matrices are represented as follows:

$$
\begin{gathered}
\Gamma_{0}\left(\tau_{k}^{\prime}\right)=\Gamma_{h / 2}+\Gamma_{\Delta}\left(\tau_{k}^{\prime}\right), \quad \Gamma_{1}\left(\tau_{k}^{\prime}\right)=\Gamma_{h / 2}^{\prime}-\Gamma_{\Delta}\left(\tau_{k}^{\prime}\right) \\
-\frac{\Gamma_{h}}{2} \leq \Gamma_{\Delta} \leq \frac{\Gamma_{h}}{2},
\end{gathered}
$$

where

$$
\begin{gathered}
\Gamma_{h / 2}=\int_{0}^{h / 2} e^{A s} B d s, \quad \Gamma_{h / 2}^{\prime}=\int_{h / 2}^{h} e^{A s} B d s \\
\Gamma_{h}=\int_{0}^{h} e^{A s} B d s .
\end{gathered}
$$

Then it is possible to represent these discretized input matrices as uncertainty structures by defin$\operatorname{ing} \Gamma_{\Delta}\left(\tau_{k}^{\prime}\right) \equiv E \Delta\left(\tau_{k}^{\prime}\right) F$ as follows:

$$
\begin{gathered}
\Gamma_{0}\left(\tau_{k}^{\prime}\right)=\Gamma_{h / 2}+E \Delta\left(\tau_{k}^{\prime}\right) F \\
\Gamma_{1}\left(\tau_{k}^{\prime}\right)=\Gamma_{h / 2}^{\prime}+(-E) \Delta\left(\tau_{k}^{\prime}\right) F,
\end{gathered}
$$

where

$$
\left\|\Delta\left(\tau_{k}^{\prime}\right)\right\| \leq 1, \quad E=\frac{1}{2} I, \quad F=\Gamma_{h} .
$$

Based on the above uncertainty structure, we can account for the delay $\tau_{k}^{\prime}$ smaller than the sampling period as follow:

Theorem 3. Suppose that there exist $P(i)>$ $0, K(i)(i=1, \ldots, N), Q, \mathcal{X}, \mathcal{Y}, \mathcal{Z}$, and scalars $e_{1}, e_{2}, e_{3}$ such that

$$
\left[\begin{array}{cccccc}
\Sigma_{11} & -\mathcal{Y} & \tilde{\Phi}^{T} & \Sigma_{41}^{T} & 0 & 0 \\
-\mathcal{Y}^{T} & -Q & \Sigma_{32}^{T} & \Sigma_{42}^{T} & \Sigma_{52}^{T} & \Sigma_{62}^{T} \\
\tilde{\Phi} & \Sigma_{32} & \Sigma_{33} & e_{3} \tilde{E} \tilde{E}^{T} & 0 & 0 \\
\Sigma_{41} & \bar{\Sigma}_{42} & e_{3} \tilde{E} \tilde{E}^{T} & \Sigma_{44} & 0 & 0 \\
0 & \Sigma_{52} & 0 & 0 & -e_{1} I & -e_{3} I \\
0 & \Sigma_{62} & 0 & 0 & -e_{3} I & -e_{2} I
\end{array}\right]<0
$$

and

$$
\left[\begin{array}{cc}
\mathcal{X} & \mathcal{Y} \\
\mathcal{Y}^{T} & \mathcal{Z}
\end{array}\right] \geq 0
$$

where

$$
\begin{aligned}
& \Sigma_{32}=\tilde{\Gamma}_{T / 2} \tilde{K}(i), \quad \Sigma_{33}=-\bar{P}^{-1}(i)+e_{1} \tilde{E} \tilde{E}^{T} \\
& \Sigma_{42}=(\bar{d}-1) \tilde{\Gamma}_{T / 2} \tilde{K}(i), \quad \Sigma_{44}=-(\bar{d}-1) \mathcal{Z}^{-1}+e_{2} \tilde{E} \tilde{E}^{T} \\
& \Sigma_{52}=\tilde{F} \tilde{K}(i), \quad \Sigma_{62}=(\bar{d}-1) \tilde{F} \tilde{K}(i) \\
& \tilde{\Gamma}_{T / 2}=\left[\begin{array}{cc}
\Gamma_{T / 2} & \Gamma_{T / 2}^{\prime} \\
0 & 0
\end{array}\right], \quad \tilde{E}=\left[\begin{array}{c}
E \\
0
\end{array}\right] \\
& \tilde{F}=\left[\begin{array}{ll}
F-F
\end{array}\right], \quad \tilde{K}(i)=\left[\begin{array}{cc}
K(i) & 0 \\
0 & K(i)
\end{array}\right] .
\end{aligned}
$$

Then the closed-loop system (6) with uncertainty structures (21)-(22) is asymptotically stable for the time-varying network induced delay $\tau_{k}$ satisfying $0 \leq \tau_{k} \leq\left(d_{k}-1\right) h+\tau_{k}^{\prime}$, and $\tau_{k}^{\prime}$ is a time-varying value with $0<\tau_{k}^{\prime} \leq h$, where $h$ is the sampling period, and $d_{k} \geq 1$ is integer valued Markov states.

Proof : Replace $\Gamma_{0}$ and $\Gamma_{1}$ in Theorem 1 with $\left(\Gamma_{h / 2}+E \Delta\left(\tau_{k}^{\prime}\right) F\right)$ and $\left(\Gamma_{h / 2}^{\prime}+(-E) \Delta\left(\tau_{k}^{\prime}\right) F\right)$, respectively. And multiply both sides of the resulting inequality by the vector $x_{i}(i=1,2,3,4)$, which yields the following condition

$$
\left[\begin{array}{l}
x_{1} \\
x_{2} \\
x_{3} \\
x_{4}
\end{array}\right]^{T}\left[\begin{array}{cccc}
\Sigma_{11} & -\mathcal{Y} & \tilde{\Phi}^{T} & \Sigma_{41}^{T} \\
(*) & -Q & (2,3) & (2,4) \\
(*) & (*) & -\bar{P}^{-1}(i) & 0 \\
(*) & (*) & (*) & -(\bar{d}-1) \mathcal{Z}^{-1}
\end{array}\right]\left[\begin{array}{l}
x_{1} \\
x_{2} \\
x_{3} \\
x_{4}
\end{array}\right]<0
$$

where $(2,3)=\tilde{K}^{T}(i)\left[\tilde{\Gamma}_{T / 2}+\tilde{E} \Delta\left(\tau_{k}^{\prime}\right) \tilde{F}\right]^{T},(2,4)=$ $(\bar{d}-1) \tilde{K}^{T}(i)\left[\tilde{\Gamma}_{T / 2}+\tilde{E} \Delta\left(\tau_{k}^{\prime}\right) \tilde{F}\right]^{T}$. Let us define new variables as

$$
p_{1} \equiv \Delta^{T}\left(\tau_{k}^{\prime}\right) \tilde{E}^{T} x_{3}, p_{2} \equiv \Delta^{T}\left(\tau_{k}^{\prime}\right) \tilde{E}^{T} x_{4},
$$

then we have

$\left[\begin{array}{l}x_{1} \\ x_{2} \\ x_{3} \\ x_{4} \\ p_{1} \\ p_{2}\end{array}\right]^{T}\left[\begin{array}{cccccc}\Sigma_{11} & -\mathcal{Y} & \tilde{\Phi}^{T} & \Sigma_{41}^{T} & 0 & 0 \\ (*) & -Q & (2,3) & (2,4) & (2,5) & (2,6) \\ (*) & (*) & (3,3) & 0 & 0 & 0 \\ (*) & (*) & (*) & (4,4) & 0 & 0 \\ (*) & (*) & (*) & (*) & 0 & 0 \\ (*) & (*) & (*) & (*) & (*) & 0\end{array}\right]\left[\begin{array}{l}x_{1} \\ x_{2} \\ x_{3} \\ x_{4} \\ p_{1} \\ p_{2}\end{array}\right]<0$,

and

$$
\begin{aligned}
& {\left[\begin{array}{ll}
p_{1}^{T} & p_{2}^{T}
\end{array}\right]\left[\begin{array}{ll}
e_{1} I & e_{3} I \\
e_{3} I & e_{2} I
\end{array}\right]\left[\begin{array}{l}
p_{1} \\
p_{2}
\end{array}\right]} \\
& <\left[\begin{array}{ll}
x_{3}^{T} \tilde{E} & x_{4}^{T} \tilde{E}
\end{array}\right]\left[\begin{array}{ll}
e_{1} I & e_{3} I \\
e_{3} I & e_{2} I
\end{array}\right]\left[\begin{array}{l}
\tilde{E}^{T} x_{3} \\
\tilde{E}^{T} x_{4}
\end{array}\right], \\
& {\left[\begin{array}{ll}
e_{1} I & e_{3} I \\
e_{3} I & e_{2} I
\end{array}\right]>0}
\end{aligned}
$$


where $(2,3)=\tilde{K}^{T}(i) \tilde{\Gamma}_{T / 2}^{T},(2,4)=(\bar{d}-1) \tilde{K}^{T}(i) \tilde{\Gamma}_{T / 2}^{T}$, $(2,5)=\tilde{K}^{T}(i) \tilde{F}^{T},(2,6)=(\bar{d}-1) \tilde{K}^{T}(i) \tilde{F}^{T},(3,3)=$ $-\bar{P}^{-1}(i),(4,4)=-(\bar{d}-1) \mathcal{Z}^{-1}$. The above inequalities can be unified to $(24)$ by using the $\mathcal{S}$-procedure (Boyd et al., 1994).

In Theorem 2, the inequality (24) is not of a conventional LMI but a BMI(Bilinear matrix inequality) due to $\bar{P}^{-1}(i)$ and $\mathcal{Z}^{-1}$ terms. So it needs a linearization technique (Ghaoui et al., 1997) as follows:

Lemma 4. (Cone-complement linearization) The nonlinear matrix inequality problems (24) and (25) can be approximated by a cone complement linearization as follows:

Find $P(i)>0, M(i)>0, K(i)$ for $i=1, \ldots, N$, and $Q, \mathcal{N}, \mathcal{X}, \mathcal{Y}, \mathcal{Z}$ and scalars $e_{1}, e_{2}, e_{3}$ such that

$$
\text { Minimize } \operatorname{tr}\left\{\sum_{i=1}^{N} \bar{P}(i) M(i)+\mathcal{Z} \mathcal{N}\right\}
$$

subject to

$$
\left[\begin{array}{cccccc}
\Sigma_{11} & -\mathcal{Y} & \tilde{\Phi}^{T} & \Sigma_{41}^{T} & 0 & 0 \\
-\mathcal{Y}^{T} & -Q & \Sigma_{32}^{T} & \bar{\Sigma}_{42}^{T} & \Sigma_{52}^{T} & \Sigma_{52}^{T} \\
\tilde{\Phi} & \Sigma_{32} & \bar{\Sigma}_{33} & e_{3} \tilde{E} \tilde{E}^{T} & 0 & 0 \\
\Sigma_{41} & \bar{\Sigma}_{42} & e_{3} \tilde{E} \tilde{E}^{T} & \bar{\Sigma}_{44} & 0 & 0 \\
0 & \Sigma_{52} & 0 & 0 & -e_{1} I & -e_{3} I \\
0 & \Sigma_{62} & 0 & 0 & -e_{3} I & -e_{2} I
\end{array}\right]<0
$$$$
\left[\begin{array}{cc}
\mathcal{X} & \mathcal{Y} \\
\mathcal{Y}^{T} & \mathcal{Z}
\end{array}\right] \geq 0
$$

and

$$
\left[\begin{array}{cc}
\bar{P}(i) & I_{2 n \times 2 n} \\
I_{2 n \times 2 n} & M(i)
\end{array}\right] \geq 0,\left[\begin{array}{cc}
\mathcal{Z} & I_{2 n \times 2 n} \\
I_{2 n \times 2 n} & \mathcal{N}
\end{array}\right] \geq 0,
$$

where

$$
\begin{aligned}
& \bar{\Sigma}_{33}=-M(i)+e_{1} \tilde{E} \tilde{E}^{T} \\
& \bar{\Sigma}_{44}=-(\bar{d}-1) \mathcal{N}+e_{2} \tilde{E} \tilde{E}^{T} .
\end{aligned}
$$

In Lemma 2, we have proposed the nonlinear minimization problem involving LMI conditions instead of the original non-convex feasibility problem of Theorem 2. Note that, although it is still impossible to always find the global optimal solution, it is easier to solve the proposed nonlinear minimization problem than the original nonconvex feasibility problem.

\section{NUMERICAL EXAMPLE}

To exemplify the performance of the proposed method in this paper, let us consider a simple unstable system as follows:

$$
\dot{x}(t)=\left[\begin{array}{cc}
0 & 1 \\
1 & -2
\end{array}\right] x(t)+\left[\begin{array}{l}
0 \\
1
\end{array}\right] u(t)
$$

The above system has poles at $s=-2.414$ and $s=$ 0.414. As sampling the continuous system with the period $h=0.01$, the non-delayed discrete-time system is given as follows:

$$
x(k+1)=\left[\begin{array}{cc}
1 & 0.0099 \\
0.0099 & 0.9802
\end{array}\right] x(k)+\left[\begin{array}{c}
0 \\
0.0099
\end{array}\right] u(k) .
$$

A compensation of network delayed system can be designed by using the proposed controller. Let us consider a network delayed system with three Markov stated chains

$$
\begin{aligned}
& \tau_{k}=\left(d_{k}-1\right) h+\tau_{k}^{\prime}, \text { and } u(t) \leftarrow u\left(t-\tau_{k}\right) \\
& \text { State 1: } d_{1}=1, \text { i.e., } \tau_{1}=\tau_{1}^{\prime}, \\
& \text { State } 2: d_{2}=5 \text {, i.e., } \tau_{2}=4 h+\tau_{2}^{\prime} \\
& \text { State 3: } d_{3}=10 \text {, i.e., } \tau_{3}=9 h+\tau_{3}^{\prime} .
\end{aligned}
$$

And the state transition matrix of those Markov chains is given by

$$
P_{t r}=\left[\begin{array}{ccc}
0.6 & 0.2 & 0.2 \\
0.2 & 0.7 & 0.1 \\
0 & 0.3 & 0.7
\end{array}\right]
$$

To solve the suboptimal problem in Lemma 2, we introduce an iterative algorithm as follows:

1. Find a feasible set $(P(i), M(i), Q, \mathcal{N}, \mathcal{X}, \mathcal{Y}$, $\left.\mathcal{Z}, K(i), e_{1}, e_{2}, e_{3}\right)$ satisfying (26)-(30). Set $n=0$.

2. Solve the following LMI problem for the variables $(P(i), M(i), Q, \mathcal{N}, \mathcal{X}, \mathcal{Y}, \mathcal{Z}, K(i)$, $\left.e_{1}, e_{2}, e_{3}\right):$ Minimize $\operatorname{tr}\left\{\sum_{i=1}^{N(=3)}\left[\bar{P}_{n}(i) M(i)\right.\right.$ $\left.\left.+M_{n}(i) \bar{P}(i)\right]+\mathcal{Z}_{n} \mathcal{N}+\mathcal{N}_{n} \mathcal{Z}\right\}$ subject to $(26)$ (30) .

3. Set $\bar{P}_{n+1}(i)=\bar{P}(i), M_{n+1}(i)=M(i)$, $\mathcal{Z}_{n+1}=\mathcal{Z}$ and $\mathcal{N}_{n+1}=\mathcal{N}$.

4. If the stopping condition (24) is satisfied, then exit. Otherwise, set $n=n+1$ and go to Step 2.

Using this iterative algorithm, we can design a discrete-time delay-dependent controller for the networked control system such that the asymptotic stability is satisfied. It is calculated by using the software LMI toolbox in MATLAB (Gahinet et al., 1995). The proposed controller is derived as follows:

$$
\begin{aligned}
& K_{1}=[-1.9053-0.3340] \text { for } d_{1}=1 \\
& K_{2}=[-1.9351-0.3393] \text { for } d_{2}=5 \\
& K_{3}=\left[\begin{array}{ll}
-1.9059-0.3389
\end{array}\right] \text { for } d_{3}=10 \text {. }
\end{aligned}
$$

Note that the network is assumed to have timevarying delays even longer than the sampling period as shown in Fig.2, which cannot have been handled by existing methods. However, the compensation method proposed in the current paper 


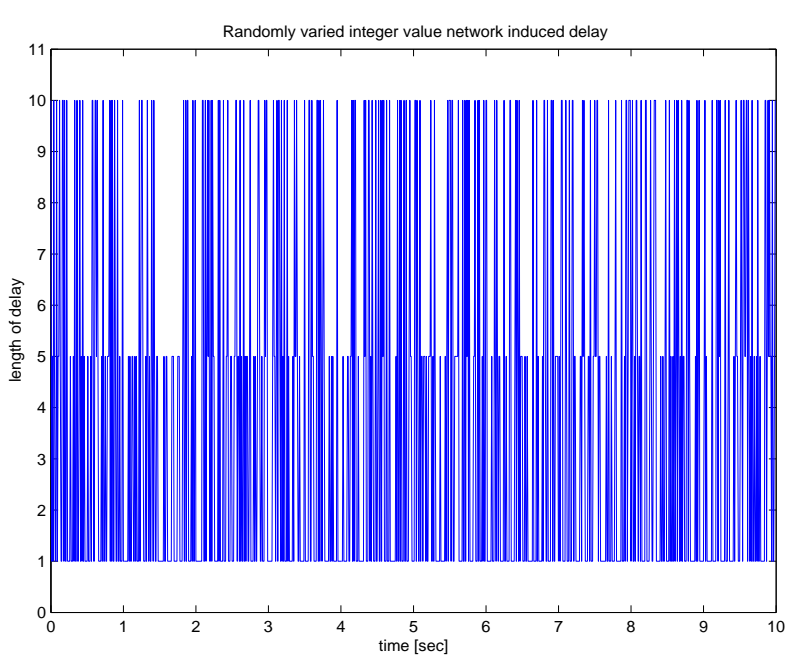

Fig. 2. Time-varying delays in the NCS

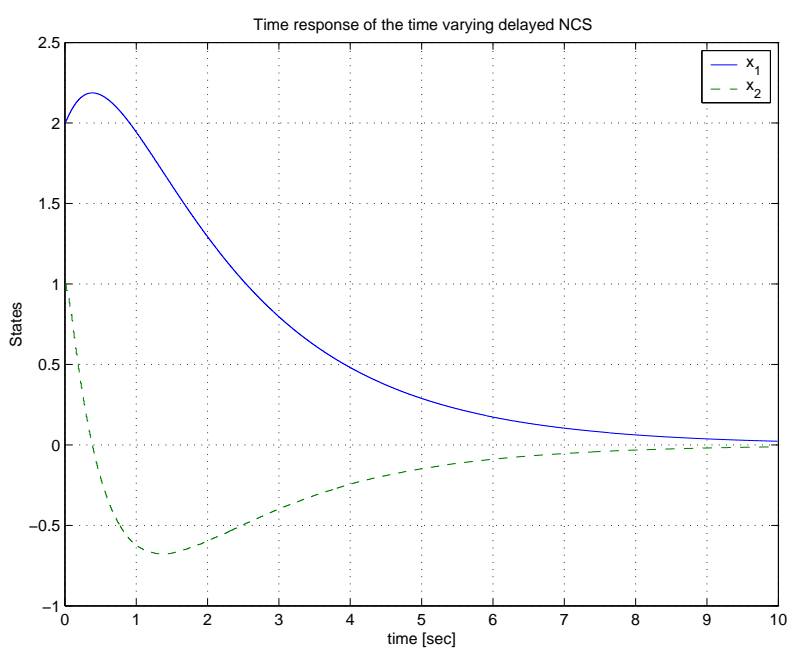

Fig. 3. State trajectories of the NCS with timevarying delays

can handle even this case, and the simulation results are shown in Fig.3. These results have shown that the proposed controller is to stabilize the unstable system with some time-varying delays, which is represented by a Markov chain. It is also noted that the proposed method is to be applied to the networked control systems with any kind of delay shorter and/or longer than the sampling time. Thus the proposed control algorithm is suitable for applications to the networked control systems.

\section{CONCLUSION}

An LMI-based delay-dependent controller has been proposed for NCS with time-varying networkinduced delays. The delays are described by a Markov chain to account for the time-varying property and the uncertainty. Using this description in the current paper, a delay-dependent controller is proposed to stabilize the networked control systems with time-varying delays. It is noted that the proposed method can account for the time-varying delays even longer than the sampling time. In order to show effectiveness of the proposed control scheme, some simulations are performed to be applied to a numerical system with the network induced delay and show that the proposed method works well.

Applications of the suggested control method to real plants requires further works. The proposed compensation method is based on the state feedback control law, and extension to the output feedback control problem falls into future works.

\section{REFERENCES}

Åström, K.J. (1997). Computer-controlled Systems (3rd Ed.). Prentice Hall Inc.

Boukas, E.K. and Z.K. Liu (2001). Roubst $h_{\infty}$ control of discrete-time markovian jump linear systems with mode-dependent timedelays. IEEE Trans. Automatic Control.

Boyd, S., L.E. Ghaoui, E. Feron and V. Balakrishnan (1994). Linear Matrix Inequalities in Systems and Control Theory. SIAM.

Gahinet, P., A. Nemirovski, A.J. Laub and M. Chilali (1995). LMI Control Toolbox for use with MATLAB. The MathWorks, Inc.

Ghaoui, L. El, F. Oustry and M. Ait Rami (1997). A cone complementarity linearization algorithms for static output-feedback and related problems. IEEE Trans. Automatic Control.

Halevi, H. and A. Ray (1988). Integrated communication and control systems: Part 1 - analysis. Journal of Dynamic Systems, Measurement, and Control.

Lee, Y.S., Y.S. Moon and W. H. Kwon (2001). Delay-dependent robust $\mathcal{H}_{\infty}$ control for uncertain systems with time-varying statedelay. Proc. of 40th IEEE CDC. pp. 32083213.

Luck, R. and A. Ray (1990). An observer-based compensator for distributed delays. Automat$i c a$.

Mariton, M. (1990). Jump Linear Systems in Automatic Control. Marcel Dekker, Inc.

Nilsson, J. and Bo Bernhardsson (1997). LQG control over a markov communication network. Proc. of 36th IEEE CDC. pp. 45864591.

Yoo, H.J., H.S. Ryu, K.S. Yoo and O. K. Kwon (2002). Compensation of networked control systems using lmi-based delay-dependent optimization method. Proc. of 41th SICE. pp. 1104-1109.

Zhang, W., M. Branicky and S. Phillips (2001). Stability of networked control systems. IEEE Control System Magazine. 